\title{
Anisotropic Properties of Directed Energy Deposition (DED)-Processed Ti-6Al-4V
}

\author{
Sarah Wolff ${ }^{*}$, Taekyung Lee ${ }^{1}$, Eric Faierson ${ }^{2}$, Kornel Ehmann ${ }^{1}$ and Jian \\ $\mathrm{Cao}^{1 \dagger}$ \\ ${ }^{1}$ Northwestern University, Evanston, IL, U.S.A. \\ ${ }^{2}$ Quad City Manufacturing Laboratory, Rock Island, IL, U.S.A. \\ sarahwolff@u.northwestern.edu, jcao@northwestern.edu
}

\begin{abstract}
Directed Energy Deposited (DED) Ti-6Al-4V components and prototypes are quickly growing inprevalence in aerospace and biomedical industries for their increased strength and fast processing time. However, one of the remaining challenges in DED processes, particularly Laser Engineered Net Shaping (LENS), is the characterization of the inherent anisotropy in material properties. Anisotropy in microstructure, porosity and mechanical behavior arises due to unique material thermal histories during processing. Understanding anisotropy in additive manufacturing can lead to refined process parameters, characterization methods, material and thermal modeling as well as improved mechanical properties. This paper investigates the anisotropic mechanical properties, specifically ultimate tensile strength, of LENS-processed Ti-6Al-4V and how these properties depend on geometry and direction of build processing. Mechanical properties were found to be most desirable when the tensile orientation is orthogonal to the build direction and parallel to the scan direction as well as closer to the center of a fully dense component. This study investigates microstructure through X-ray diffraction, fractography and porosity shape and connectivity analysis. Models that predict mechanical behavior based on processing details are in development. There is potential to achieve desirable mechanical properties through melt pool and thermal controls for high-strength and energy efficient materials by predicting mechanical properties from process parameters.
\end{abstract}

Keywords:additive manufacturing, Directed Energy Deposition (DED), Laser Engineered Net Shaping (LENS), Ti-6Al-4V, titanium alloys, anisotropy, mechanical behavior

\footnotetext{
${ }^{*}$ Corresponding Author
}

${ }^{\dagger}$ Corresponding Author

(c) 2016. This manuscript version is made available under the Elsevier user license 


\section{Introduction}

Materials pose the largest limitation to the development of additive manufacturing (AM) with a demand for better and less expensive feed stock powder materials and a need for better understanding and modeling of how materials solidify and fuse during such a complex process. Ti-6Al-4V is a highstrength and light-weight titanium alloy that is widespread in aerospace and biomedical applications, such as dental implants [1]. Ti-6Al-4V(grade 5) is generally cast with a density of $4.43 \mathrm{~g} / \mathrm{cc}$, an ultimate tensile strength (UTS) of $976 \mathrm{MPa}$, a yield strength of $847 \mathrm{MPa}$ and elongation of $5.1 \%$, whereas annealed Ti-6Al-4V has a UTS between 895 and $930 \mathrm{MPa}$, yield strength between 825 and $869 \mathrm{MPa}$, a Young's modulus between 110 and $114 \mathrm{GPa}$ and elongation between 6 and 10\% [1].Directed energy deposition is a promising additive manufacturing process to build Ti-6Al-4V components with even higher strengths and toughness, but the process also introduces an anisotropy of the components [2]. Understanding the material's anisotropy and other characteristics is crucial to developing optimal materials and the processes for additive manufacturing technologies and their applications. More specific aims include the characterization of the material and the overall influences of: location relative to the surface of a component, orientation in the component relative to the build and scan directions, and laser processing power as well as an in-depth characterization of the pores in the various orientations and components processed with various laser powers. Future work includes predicting porosity, microstructure and properties of future DED-processed Ti-6Al-4V and other metallic components.

\subsection{Directed Energy Deposition (DED) and Laser Engineered Net Shaping (LENS)}

According to ASTM F2792-12a, Directed Energy Deposition (DED) is an AM process "in which focused thermal energy is used to fuse materials by melting as they are being deposited" with an energy source, such as a laser, electron beam or plasma arc is "focused to melt the materials as they are being deposited" [3]. Laser Engineered Net Shaping (LENS) is a type of DED process that builds layer-by-layer components from .stl files by means of a moving molten pool where a laser beam melts metallic powders at the focal point on a substrate. The metal powder flows onto the focal spot from four coaxial nozzles surrounding the laser. The laser head typically has four degrees of freedom, where the lateral directions allow the laser beam to fuse metallic powders together to form a single layer. Moving the laser head in the positive $\mathrm{Z}$ direction allows additional layers to fuse with the subsequent layer. During the process, inert gas flows into the system to shield the powders and the work piece from atmospheric gases and oxidation [4].

The benefits that DED may pose over other AM processes include the ability to build net-shape, fully dense components, flexibility in process parameters, the ability to build materials with high melting points, such as titanium alloys, and resulting mechanical properties that are similar or superior to those of conventionally-processed materials [5]. However, the DED process also poses several challenges, such as limitations in building complex shapes, efficient powder delivery and most notably, control of material properties. This is because a morphology of the solidification structure arises from the high thermal gradients during the process. The relationship between process, structure and properties is evident with DED-processed materials; the combination of laser power, scan speed, powder flow rate, and molten pool size result in a unique heat transfer problem that contributes to a morphology of porosity and in strength anisotropy [6].

Gaseous porosity, voids and undesirable phase composition in the microstructure of LENSprocessed metal components can result in decreased strength and overall degradation of mechanical properties. Two types of porosity have been observed in LENS-processed metallic components and are 
sensitive to process parameters. Interlayer pores form at the interface between adjacent layers and intra-layer pores form within the mass of the layer. Interlayer porosity is caused by a discrepancy in temperature distribution in the deposited melted power, including the unwanted presence of un-melted powder particles that flow to the underlying, colder layers.

\subsection{DED-Processed Ti-6Al-4V and Anisotropy}

A few previous studies have shown strength anisotropy as a result of the relative orientation to the build and scan directions, with yield strengths in the $\mathrm{X}$ and $\mathrm{Y}$ (scan) directions higher than that in the $\mathrm{Z}$ (build) direction due to lack of fusion of powders between layers [2,7-9].

Several studies have investigated the tensile behavior of LENS-processed, wrought and cast Ti$6 \mathrm{Al}-4 \mathrm{~V}$ with results showing that porosity due to lack of fusion, as opposed to porosity due to entrapped gas, had a significant influence on the anisotropy of mechanical properties [2,7-13].One study calculated the amount of anisotropy that each orientation of the Ti-6Al-4V LENS build exhibited, with tensile strength anisotropy as much as about $12.5 \%$ and the anisotropy in yield strength was over $40 \%$ with elongation significantly lower than that of wrought titanium alloys [8].They found that LENS-processed Ti-6Al-4V had comparable properties to cast titanium and that crack initiation sites during tensile testing were results of coalesced pores that nucleated during the LENS process [8]. A recent study investigated DED-processed Ti-6Al-4V tensile specimens built with a variety of orientations and locations relative to the build plate [12]. Similar to previous research, this study found competitive properties of the DED Ti-6Al-4V, with long and thin prior-beta grains growing in the build direction resulting in anisotropic tensile elongation properties with higher ductility in the transverse direction $[10,12]$. A recent study that found anisotropic mechanical behavior of LENSprocessed Ti-6Al-4V components found that inhomogeneous cooling rates throughout the part led to extensive and ordered porosity, especially in the vertical direction, which could be reduced by HIP, and lead to competitive properties and possible control of process parameters [7].

A few more studies looked more closely at the influence of process parameters [9-11,13]. One of these studies looked at DED of Ti-6Al-4V wire of $1.2 \mathrm{~mm}$ diameter to create tubular components to undergo tensile test in order to investigate microstructure and mechanical behavior [12]. Compared to the UTS and elongation at break of cast Ti-6Al-4V at $976 \mathrm{MPa}$ and 5.1\% respectively, the study found competitive UTS and elongation at break values of 929-1014 MPa and 10-20\% for the DED-processed tubular components. In addition, the differences in properties among the tubular components were due to the different process parameters that resulted in various alpha lath size and the orientation of the elongated prior beta grains, where smaller lamellae width of the alpha grains led to greater UTS [10].

Based on the literature, the overall lesson learned is that DED-processed Ti-6Al-4V components exhibit competitive properties with cast and wrought parts but also exhibit porosity and anisotropy in structure and mechanical behavior. These existing studies highlight the need to define relationships that link DED process parameters, microstructure of the resulting material, and the anisotropic mechanical behavior, which is attempted in this paper.

\section{Sample Preparation}

The experimental portion of this study illustrates the strength and elongation anisotropies in DEDprocessed Ti-6Al-4V components and how these properties change in not only orientation and laser processing power, but also location from the surface of a component due to the unique thermal histories of each layer and area of a component. Relationships among the process parameters, porosity, 
and the tension test results will be made in an attempt to predict material structures and properties based on the process parameters and later manipulate the parameters to control the thermal histories.

\subsection{LENS Processing of Ti-6Al-4V Cubic Components}

Three Ti-6Al-4V cubic components were built with a closed-loop, enclosed Sandia National Laboratories Laser Engineered Net Shaping ${ }^{\circledR}$ system housed in Quad City Manufacturing Laboratory in Rock Island, IL. The LENS system had a $1 \mathrm{~kW}$ Ytterbium continuous wave (CW) fiber optic laser with a Gaussian beam, inert gas operations, 4 axis capability, and $300 \mathrm{~mm}$ x $300 \mathrm{~mm}$ x $300 \mathrm{~mm}$ build capacity. The Ti-6Al-4V powder used ranged in diameter from 45 to $150 \mu \mathrm{m}$ with chemical composition detailed in Table 1.

\begin{tabular}{|l|l|l|l|l|l|l|l|l|}
\hline $\begin{array}{l}\text { Chemical } \\
\text { Element }\end{array}$ & $\mathrm{Ti}$ & $\mathrm{Al}$ & $\mathrm{V}$ & $\begin{array}{l}\mathrm{Fe}, \\
\max .\end{array}$ & $\begin{array}{l}\mathrm{O}_{2}, \\
\max .\end{array}$ & $\begin{array}{l}\mathrm{N}_{2}, \\
\max .\end{array}$ & $\begin{array}{l}\mathrm{C}, \\
\text { max. }\end{array}$ & $\begin{array}{l}\text { Other } \\
\text { total, } \\
\text { max. }\end{array}$ \\
\hline Wt. \% & Bal. & 6.35 & 4.00 & 0.21 & 0.06 & 0.02 & 0.01 & $<0.40$ \\
\hline
\end{tabular}

Table 1:Chemical analysis of Ti-6Al-4V powder for LENS build

The three Ti-6Al-4V cubic components had dimensions of $40 \mathrm{~mm}$ x $40 \mathrm{~mm}$ x $40 \mathrm{~mm}$ and were built on an unheated substrate plate of the same materialwithin the LENS enclosed system. The cubic components were built with the same process parameters and tool path with the exception of the input laser processing power. The laser beam had a diameter of $1.83 \mathrm{~mm}$ and set at three powers $-710 \mathrm{~W}$, $800 \mathrm{~W}$ and $940 \mathrm{~W}$, one consistent power setting for each cubic component. The laser optic scan speed was $10 \mathrm{~mm} / \mathrm{s}$ throughout the tool path. The powder deposition rate from the four nozzles surrounding the laser optic was $0.12 \mathrm{~g} / \mathrm{s}$. The tool path consisted of the laser on ON mode while alternately scanning 0 and 180 degrees within each layer with an overlap hatch spacing of each bead set at 1.25 $\mathrm{mm}$, then on OFF mode while moving the laser optic in the positive $\mathrm{Z}$ direction by the layer thickness, or $0.95 \mathrm{~mm}$. Figure 1 illustrates the coordinate system of the LENS system and a representation of the tool path used to build the three cubic components.

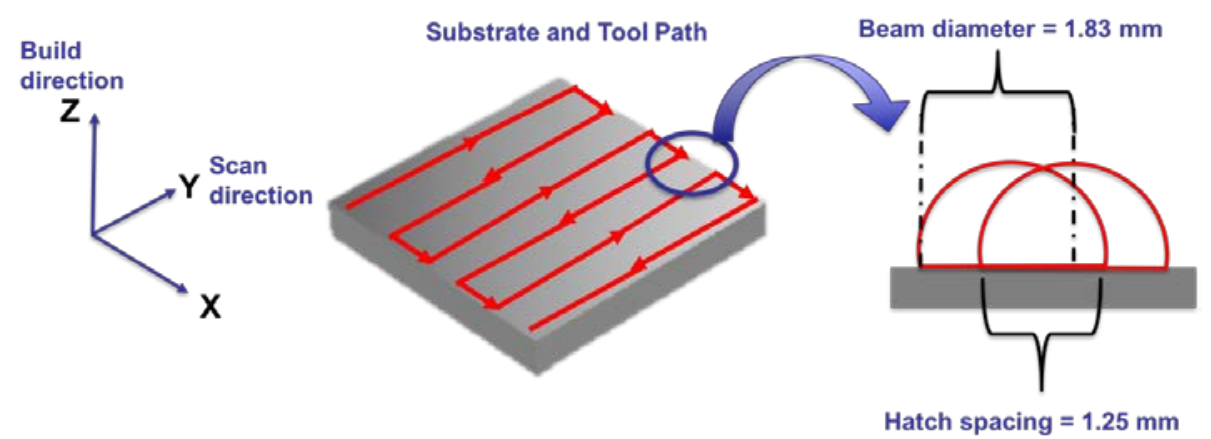

Figure 1:LENS tool path of each build on the Ti-6Al-4V substrate plate. Y denotes the scanning direction and $\mathrm{Z}$ denotes the build direction of the laser optic

\subsection{Mechanical Testing of Machined Specimens}

Mini-tensile specimens were machined by wire-EDM from the cubic in three different orientations as illustrated in Figure 2. The tensile dimensions followed ASTM E8 standards for subsized sheet tensile specimens [14] with a gauge length of $10.0 \mathrm{~mm}$, gauge width of $2.5 \mathrm{~mm}$, and thickness of $1.2 \mathrm{~mm}$. Tensile specimens with orientation (010) were machined from the XZ plane and varied in the $\mathrm{Y}$ direction and with $\mathrm{Z}$, the build direction, as their uniaxial tensile axis. Tensile specimens with orientation (001) varied in the Z direction and had $\mathrm{X}$ as their tensile axis. Tensile 
specimens with orientation (100) varied in the $\mathrm{X}$ direction and had $\mathrm{Y}$, the scan direction, as their tensile axis.Eight tensile specimens were machined in three orientations from the cubic component processed with $800 \mathrm{~W}$, resulting in 24 tensile specimens from the $800 \mathrm{~W}$ cubic component.For the 710 W cubic components, Sixspecimens in each of the (001) and (100) orientations were tested from the 710W cubic components, and six specimens from the (100) orientation was tested for the $940 \mathrm{~W}$ cubic component.The reduced number of machined specimens for the $710 \mathrm{~W}$ and $940 \mathrm{~W}$ components were due to machining costs and the fact that superior mechanical behavior had been observed in the (001) and (100) orientations. The total number from all three cubes is 42 tensile specimens.

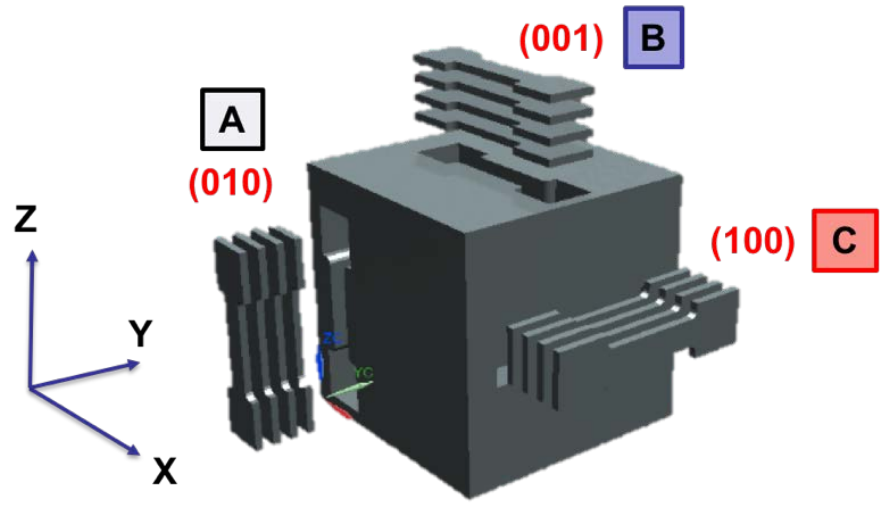

Figure 2:LENS-processed Ti-6Al-4V cubic component with tensile specimens in three orientations

The tension test load frame included a servo-hydraulic machine with $100 \mathrm{kN}$ capacity. The standard quasi-static test consisted of attaching a $10 \mathrm{~mm}$ extensometer to each tensile specimen and exerting tensile load at a strain rate of $0.25 \mathrm{~mm} / \mathrm{min}$.

\subsection{Material Characterization}

Prior to tensile testing of each specimen, bulk porosity and density values for each cubic component and later each machined tensile specimen were determined using the Archimedes method. After each tensile specimen fractured during tension, porosity and density values were determined for each half of each specimen with the Archimedes method. Each component and specimen were measured three times to obtain average values.

$\mathrm{X}$-ray diffraction (XRD) was carried out to evaluate the phase composition differences for each orientation of the $800 \mathrm{~W}$ cubic component. Scanning electron microscopy (SEM) was used to evaluate the fracture surfaces of several fractured tensile specimens in order to carry out fractography image analysis. Optical microscopy allowed for the imaging of solidification microstructure and the porosity distribution after each specimen was ground, polished and ultrasonically cleansed. The cross-sections evaluated under optical microscopy were cut from the tensile specimen grips using an aluminum silica blade.

\section{Approach for Characterizing Mechanical Properties and Pores using Generalized Mixture Rule (GMR) and J-Factor}

To establish relationships between porosity and resulting mechanical behavior in each tensile specimen, Generalized Mixture Rule (GMR) analysis was used. The GMR is fundamental composite theory that aims to mathematically define the relationship between a poly-phase structure and the 
resulting mechanical properties. For porous materials, the GMR can reflect the random nature of the solidified microstructure with porosity distribution with properties like elastic constants, yield strength, and ultimate tensile strength [15]. $J$ is a fractal parameter and numeric value that applies to isotropic materials and is controlled by the shape and distribution of the phases. $J$ is a key factor in these relationships in that $J$ captures the continuity and connectivity of the pores in the material. This fractal parameter is controlled by the shape, size distribution and spatial distribution of the pores. The general GMR equation is as follows:

$$
M_{c}^{J}=\left(1-V_{w}\right) M_{s}^{J}+V_{w} M_{w}^{J}(1)
$$

where $M_{c}$ is the mechanical property of the overall composite, $V_{w}$ is the volume of the porous phase, $M_{s}$ is the mechanical property of the bulk, single phase material, and $M_{w}$ is the mechanical property of the second phase, which in this case, is air and where the properties are equivalent to zero. Eqn. 2 applies for porous materials:

$$
\frac{M_{c}}{M_{s}}=(1-p)^{\frac{1}{J}}=V_{s}=\left(\frac{\rho_{c}}{\rho_{s}}\right)^{\frac{1}{J}}(2)
$$

where $\boldsymbol{p}$ is the porosity volume fraction in a composite, $\boldsymbol{V}_{\boldsymbol{s}}$ is the volume of the bulk, single phase material, or $\mathbf{1}-\boldsymbol{V}_{\boldsymbol{w}}, \boldsymbol{\rho}_{\boldsymbol{c}}$ is the density of the composite phase and $\boldsymbol{\rho}_{\boldsymbol{s}}$ is the density of the solid bulk phase [15]. Moreover, when the component with low porosity values, i.e., when $p$ is less than $10 \%$, the Taylor expansion of Eqn. 2 can be approximated to the following equation:

$$
\frac{M_{c}}{M_{s}}=1-\frac{1}{J} p \text { (3) }
$$

The use of curve-fitting $J$ factor in GMR relationships is to predict either the mechanical behavior or microstructure by having information of the other. Figure 3 shows the flow chart to determine the Jfactor and its link to process parameters and mechanical properties. This includes determining a thermal strain parameter that incorporates the process parameters, especially laser power, and using this thermal strain parameter to determine the ellipsoid pore dimensions.

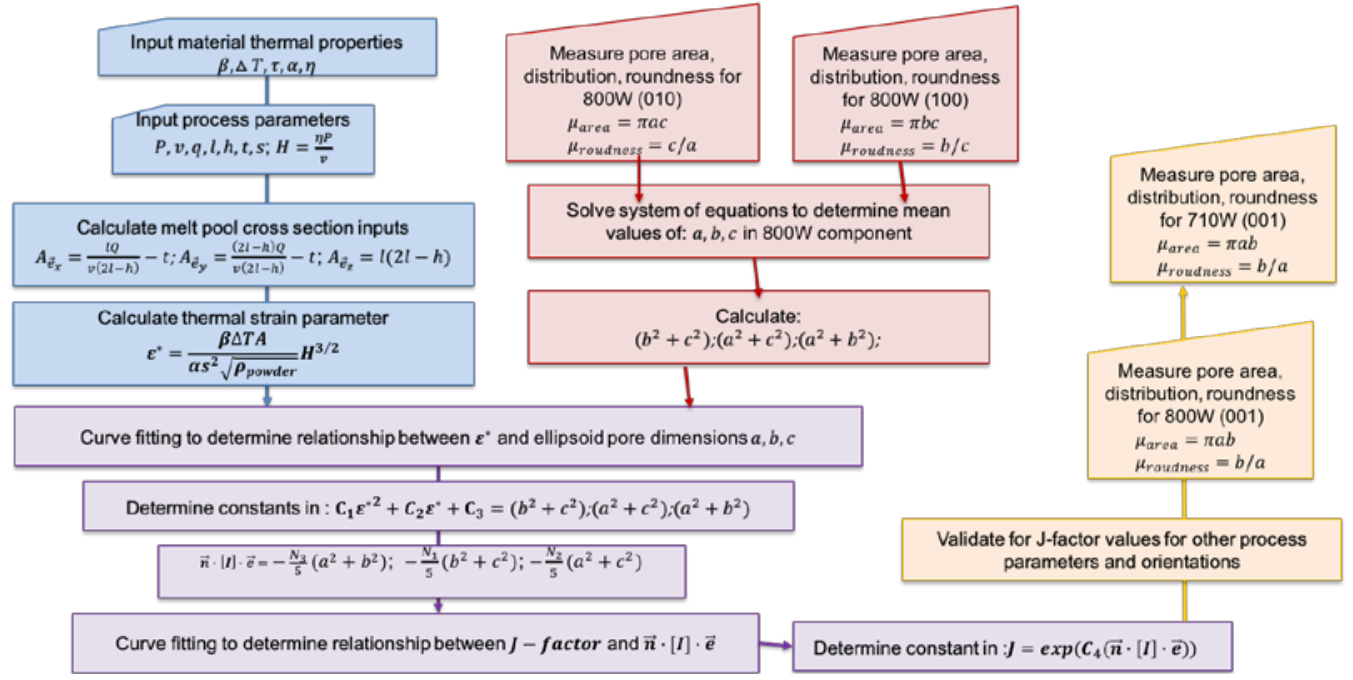

Figure 3. Flowchart to determine the J-factor for additively manufactured Ti-6Al-4V components. 


\subsection{Determination of Thermal Strain Parameter}

Our current study aims to determine the J-factor based on the mechanical test results and the porosity measurements from $2 \mathrm{D}$ optical microscopy images. The pores that are evaluated are the interlayer pores due to the lack of fusion, as they contribute to the majority of the porosity and to mechanical failure. These pores are assumed to be ellipsoids, or ellipses in 2D image analysis.

To account for the influence of process parameters, dimensional inaccuracy due to thermal distortion during the process is calculated to determine the amount of lack of fusion that would cause porosity [16]. A non-dimensional thermal strain parameter, $\varepsilon^{*}$, can be calculated based on the Buckingham $\pi$-theorem and by using process parameters as variables [16,17]. Table 2 lists the variables and their dimensions in the MLT $\theta$ system.

\begin{tabular}{|c|c|}
\hline Variable - Material Properties & Dimension \\
\hline Volumetric thermal expansion coefficient, $\beta$ & $\theta^{-1}$ \\
\hline $\begin{array}{l}\text { Temperature gradient, } \Delta T=T_{\text {liquidus }}-T_{\text {solidus }} \text {, which refer to the temperature } \\
\text { difference between the liquidus and solidus temperatures of Ti-6Al- } 4 \mathrm{~V}\end{array}$ & $\theta$ \\
\hline Characteristic time scale for solidification cooling, $\tau$ & $T$ \\
\hline $\begin{array}{l}\text { Thermal diffusivity, } \alpha=k / \rho_{\text {powder }} C_{P} \text {, where } k, \rho_{\text {powder }}, C_{P} \text { are thermal } \\
\text { conductivity, density of powder and specific heat for Ti-6Al- } 4 \mathrm{~V} \text {, respectively }\end{array}$ & $L^{2} T^{-1}$ \\
\hline Absorption coefficient, $\eta$ & Dimensionless \\
\hline Variable - Process Parameters & Dimension \\
\hline $\begin{array}{l}\text { Heat input per length, } H=\eta P / v \text { where } \eta, P, v \text { refer to the absorption } \\
\text { coefficient, laser beam power }(\mathrm{W}) \text { and the scan speed of the laser beam }(\mathrm{mm} / \mathrm{s})\end{array}$ & $M L T^{-2}$ \\
\hline $\begin{array}{l}\text { Volumetric powder flow, } Q=q / \rho_{\text {powder }} \text {, where } q \text { is the mass powder flow and } \\
\rho_{\text {powder }} \text { is the density of Ti-6Al-4V powder, which is about } 60 \% \text { of bulk Ti-6Al- } \\
4 \mathrm{~V}\end{array}$ & $L^{3} T^{-1}$ \\
\hline $\begin{array}{l}\text { Cross-sectional area, } A \text { of the ellipsoid melt pool, with volume } V=\frac{4}{3} \pi *(l) * \\
(2 l-h) *\left(\frac{Q}{v(2 l-h)}-t\right) \text {, where } l \text { is the laser beam diameter and the length of the } \\
\text { melt pool in the scan speed direction, } 2 l-h \text { is the width of the melt pool in the } \\
\text { hatch direction with } h \text { hatch spacing, and } \frac{Q}{v(2 l-h)}-t \text { the depth of the melt pool } \\
\text { withtlayer thickness }\end{array}$ & $L^{2}$ \\
\hline Width, length and height of the cubic component, $s$ & $L$ \\
\hline
\end{tabular}

Table 2: Variables used in the dimensional analysis for thermal strain parameter.

The resulting thermal strain parameter can be expressed as a function of the LENS process parameters and material thermal properties (Mukherjee, 2016):

$$
\varepsilon^{*}=\frac{\beta \Delta T A}{\alpha s^{2} \sqrt{\rho_{\text {powder }}}} H^{3 / 2} \text { (4) }
$$

The following table provides the resulting thermal strain parameters for each load axis, $\boldsymbol{e}$, in a cubic component, for the three laser processing powers. Each load axis will have a different crosssectional area for the melt pool, which will influence the weight of the parameter. The thermal strain parameter will be a weighting factor to J-factor calculations.

\begin{tabular}{|c|c|c|c|c|}
\hline $\begin{array}{c}\text { Principal load } \\
\text { axis }\end{array}$ & $\mathbf{7 1 0 ~ W}$ & $\mathbf{8 0 0 W}$ & $\mathbf{9 4 0 W}$ & $\mathrm{C}_{\mathbf{1}} \boldsymbol{\varepsilon}^{* 2}+\boldsymbol{C}_{\mathbf{2}} \boldsymbol{\varepsilon}^{*}+\mathbf{C}_{\mathbf{3}}=$ \\
\hline $\overrightarrow{\boldsymbol{e}_{\boldsymbol{x}}}$ & 0.217 & 0.260 & 0.331 & $\boldsymbol{b}^{\mathbf{2}}+\boldsymbol{c}^{\mathbf{2}}$ \\
\hline $\overrightarrow{\boldsymbol{e}_{\boldsymbol{y}}}$ & 0.286 & 0.343 & 0.436 & $\boldsymbol{a}^{\mathbf{2}}+\boldsymbol{c}^{\mathbf{2}}$ \\
\hline $\overrightarrow{\boldsymbol{e}_{\mathbf{z}}}$ & 0.543 & 0.650 & 0.827 & $\boldsymbol{a}^{\mathbf{2}}+\boldsymbol{b}^{\mathbf{2}}$ \\
\hline
\end{tabular}

Table 3: Thermal strain parameters for the various laser power and directions in $\%$. 


\subsection{Determination of the J-factor}

To properly characterize the relationship between process parameters and mechanical behavior, the size, orientation and shape of the individual pores should be captured. In order to do this, each orientation should be characterized by the normal vector to its plane, $\overrightarrow{\boldsymbol{n}}$, and the tensile test load axis, $\boldsymbol{e}$, as shown in Table 3. Moment of the LENS-processed component is calculated as a composite where the bulk material is a cube and the pores are $N$ number of aligned ellipsoids, as seen in Figure 3. The axes about which the moment of inertia is calculated is through the center of the cube, with each axis the principal axes of $\mathrm{x}, \mathrm{y}$ and $\mathrm{z}$.

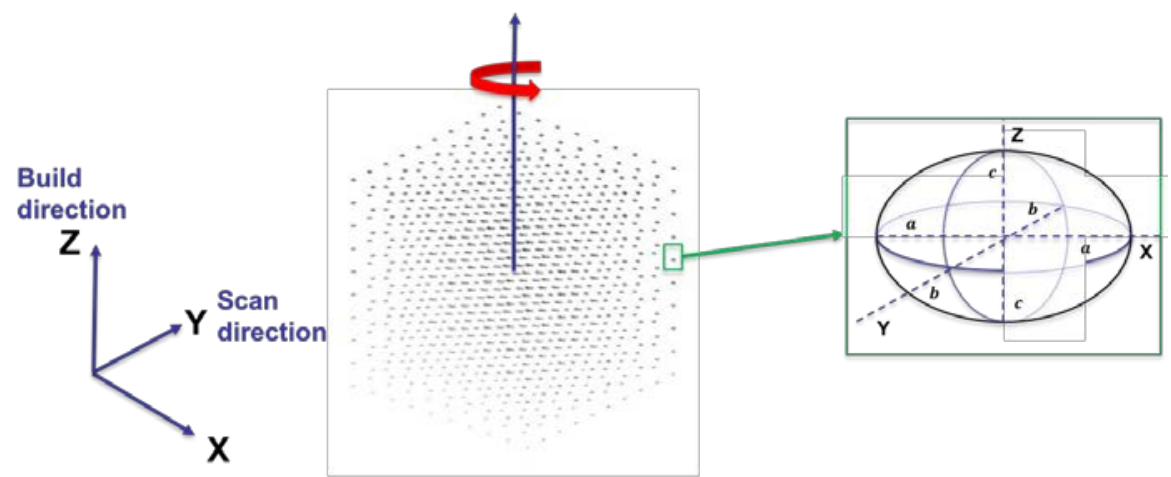

Figure 4. Visual representation of a bulk component with aligned ellipsoid pores with pore dimensions a, b, c.

The moment of inertia of the composite is expressed as a tensor. If $m$ is the mass of the Ti-6Al-4V bulk material, $a, b, c$ are the dimensions of the ellipsoid pores. $N_{1}, N_{2}, N_{3}$ are the number of pores in the $\overrightarrow{\boldsymbol{e}_{\boldsymbol{x}}} \overrightarrow{\boldsymbol{e}_{\boldsymbol{y}}}, \overrightarrow{\boldsymbol{e}_{\boldsymbol{z}}}$ load axes, respectively. $N_{1}$ is the width of the cubic component divided by the hatch spacing in the $\overrightarrow{\boldsymbol{e}_{\boldsymbol{x}}}$ direction, $N_{2}$ is the length of the cubic component divided by the hatch spacing in the $\overrightarrow{\boldsymbol{e}_{\boldsymbol{y}}}$ direction, and $N_{3}$ is the height of the cubic component divided by the hatch spacing, or in this case, the layer thickness, in the $\overrightarrow{\boldsymbol{e}_{\mathbf{z}}}$ direction.

The total moment of inertia of the porous component can be described as:

$$
\left[I_{t o t}\right]=\left[\begin{array}{ccc}
I_{x x} & I_{x y} & I_{x z} \\
I_{y x} & I_{y y} & I_{y z} \\
I_{z x} & I_{z y} & I_{z z}
\end{array}\right]=m\left[\begin{array}{ccc}
\frac{s^{2}}{6}-\frac{N_{1}}{5}\left(b^{2}+c^{2}\right) & -\frac{N_{1}}{5}\left(b^{2}+c^{2}\right) & -\frac{N_{1}}{5}\left(b^{2}+c^{2}\right) \\
-\frac{N_{2}}{5}\left(a^{2}+c^{2}\right) & \frac{s^{2}}{6}-\frac{N_{2}}{5}\left(a^{2}+c^{2}\right) & -\frac{N_{2}}{5}\left(a^{2}+c^{2}\right) \\
-\frac{N_{3}}{5}\left(a^{2}+b^{2}\right) & -\frac{N_{3}}{5}\left(a^{2}+b^{2}\right) & \frac{s^{2}}{6}-\frac{N_{3}}{5}\left(a^{2}+b^{2}\right)
\end{array}\right]
$$

Determination of the J-factor can be described in relationship in Eqn. 6:

$$
J=\exp \left(C_{4}(\overrightarrow{\boldsymbol{n}} \cdot[\boldsymbol{I}] \cdot \overrightarrow{\boldsymbol{e}})\right)
$$

The following table lists the $\overrightarrow{\boldsymbol{n}}$ and $\overrightarrow{\boldsymbol{e}}$ vectors for each orientation. The $a, b, c$ dimensions of the ellipsoid pores are determined by experimental results and by measuring distributions of the area and roundness of the pores in the tensile specimens of the varying orientations where $a>c>b$ based on experimental results, or image analyses of the micrographs. 


\begin{tabular}{|l|l|l|c|c|c|}
\hline Orientation & $\begin{array}{l}\overrightarrow{\boldsymbol{n}}, \text { vector } \\
\text { normal to } \\
\text { plane }\end{array}$ & $\begin{array}{l}\overrightarrow{\boldsymbol{e}}, \text { principal } \\
\text { load axis }\end{array}$ & $\begin{array}{l}\text { Area of } \\
\text { ellipsoid }\end{array}$ & $\begin{array}{l}\text { Roundness } \\
\text { of ellipsoid }\end{array}$ & $\overrightarrow{\boldsymbol{n}} \cdot[\boldsymbol{I}] \cdot \overrightarrow{\boldsymbol{e}}$ \\
\hline $\mathrm{A}(010)$ & {$\left[\begin{array}{lll}0 & 1 & 0\end{array}\right]$} & {$\left[\begin{array}{lll}0 & 0 & 1\end{array}\right]$} & $\pi a c$ & $c / a$ & $-\frac{N_{3}}{5}\left(a^{2}+b^{2}\right)$ \\
\hline $\mathrm{B}(001)$ & {$\left[\begin{array}{lll}0 & 0 & 1\end{array}\right]$} & {$\left[\begin{array}{lll}1 & 0 & 0\end{array}\right]$} & $\pi a b$ & $b / a$ & $-\frac{N_{1}}{5}\left(b^{2}+c^{2}\right)$ \\
\hline $\mathrm{C}(100)$ & {$\left[\begin{array}{lll}1 & 0 & 0\end{array}\right]$} & {$\left[\begin{array}{lll}0 & 1 & 0\end{array}\right]$} & $\pi b c$ & $b / c$ & $-\frac{N_{2}}{5}\left(a^{2}+c^{2}\right)$ \\
\hline
\end{tabular}

Table 4. Defining each orientation with unit vector normal to the plane and principal load axis.

A roundness value of 1.0 also indicates a perfect circle. Roundness values range from 0 to 1 . Area and roundness data for each specimen were interpreted as Weibull distributions, $f_{\text {area }}(u)$ and $f_{\text {roundness }}(v)$. Each Weibull distribution was defined by shape parameters, $c_{\text {area }}, d_{\text {area }}, c_{\text {roundness }}$ and $c_{\text {roundness }}$. The Weibull probability distribution functions (pdf) for areaand roundness are defined by:

$$
\begin{aligned}
& f_{\text {area }}\left(u ; c_{\text {area }}, d_{\text {area }}\right)= \begin{cases}\frac{d_{\text {area }}}{c_{\text {area }}}\left(\frac{u}{c_{\text {area }}}\right)^{d_{\text {area }}-1} e^{-\left(\frac{u}{c_{\text {area }}}\right)^{d_{\text {area }}},}, x \geq 0 \\
0, x<0\end{cases} \\
& f_{R}\left(v ; c_{\text {roundness }}, d_{\text {roudness }}\right)= \begin{cases}\frac{d_{\text {roudness }}}{c_{\text {roundness }}}\left(\frac{v}{c_{\text {roundness }}}\right)^{d_{\text {roudness }}-1} e^{-\left(\frac{v}{c_{\text {roudness }}}\right)^{d_{\text {roudness }}},}, x \geq 0(\mathbf{8}) \\
0, x<0\end{cases}
\end{aligned}
$$

The dimensions of $a, b, c$ for the ellipsoid pores will provide inputs into the moment of inertia and finally the J-fator. GMR analysis will lead to the development of a relationship between physical characteristics of the pores - the shape and the size distribution - to the J-factor for each case of power and orientation and ultimately, processing parameters so that mechanical behavior can be predicted.

\section{Experimental Results and Discussion}

Experimental results from various methods of material characterization were used to establish relationships among the many aspects of the DED-processed builds, including porosity, phase composition and mechanical behavior.

Three tiers of analysis were conducted: the influence of the location of the tensile specimen relative to the surface of the cubic component on mechanical behavior; influence of orientation ((010), (100), or (001)) of the tensile specimen on mechanical behavior; and the influence of laser processing power of the tensile specimens on mechanical behavior.

\subsection{Influence of Tensile Specimen Location Relative to the Surface of the Cubic Component}

For each orientation and cubic component, each tensile specimen was located in a unique position within the cube relative to the surface. This is crucial because each unique position within a component undergoes a distinct cooling rate - tensile specimens located near the surface of a component undergo more convection and radiation of heat to the surrounding air and shield gas, whereas material closer to the core of the component experiences re-melting and conduction. Therefore, mechanical behavior changes with the location of the tensile specimen relative to the surface of the cubic component, or with the cooling rate of any unique position. Figure 3 shows how 
mechanical behavior changes within the (010) orientation in the $800 \mathrm{~W}$ processed cubic component where each curve represents the distance of the tensile axis of the specimen to the surface of the cube.

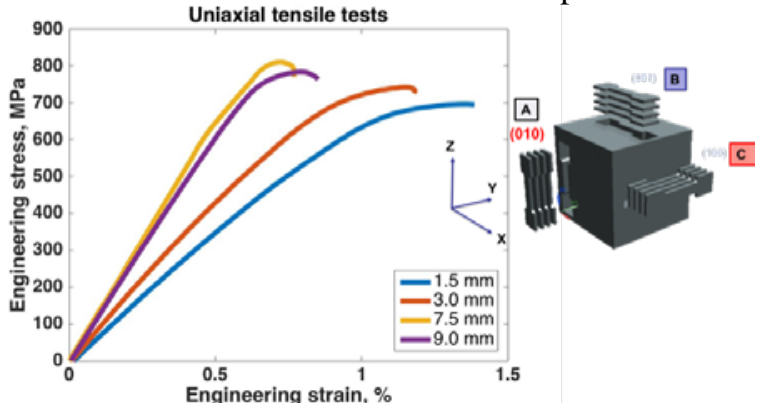

Figure 5:Tensile behavior of $800 \mathrm{~W}$ (010) specimens at various distances from cubic component surface

Based on Figure 3, the tensile specimens increase in strength as the distance from the surface of the cubic component increases, or as the tensile specimens near the core, re-melted area of the cubic in the Y scanning direction. Specimens in the (010) orientation have the greatest thermal residual stresses as each specimen has a large gradient of cooling rate, due to the re-melting at the bottom and the convection and radiation near the top of the specimen. However, specimens closer to the core fail at lower elongation values and have greater Young's moduli, implying that embrittlement occurs closer to re-melted and conductive areas for the (010) orientation. Figures 4 and 5 show the influence of location relative to the surface on mechanical behavior for orientations (001) and (100).

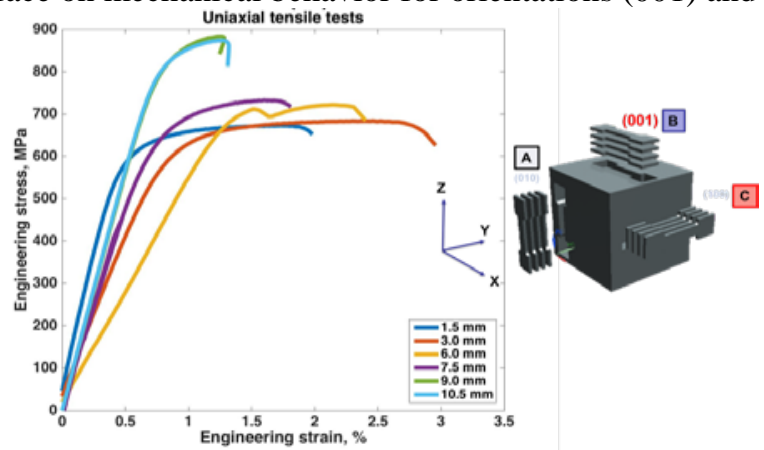

Figure 6:Tensile behavior of $800 \mathrm{~W}$ (001) specimens at various distances from cubic component surface

Tensile specimens in the (001) orientation also increase in strength from the surface to the core of the block in the negative $\mathrm{Z}$ direction, or from the top of the build to the central layers of the cubic component. Similarly to the (010) direction of the $800 \mathrm{~W}$ cube, the tensile specimens exhibit greater embrittlement and strength with an increase in distance from the surface of the cube. The Young's modulus decreases until $6 \mathrm{~mm}$ and increases from $7.5 \mathrm{~mm}$ to the center of the component. This may due to the alignment of pores that coalesce at the layer interface with the tensile axis as well as the difference in cooling rate. However, elongation of the tensile specimens are about twice the elongation values of the tensile specimens in the (010) direction. The tensile specimens in the (100) direction exhibit superior properties as compared to the specimens in the (010) and (001) directions. 


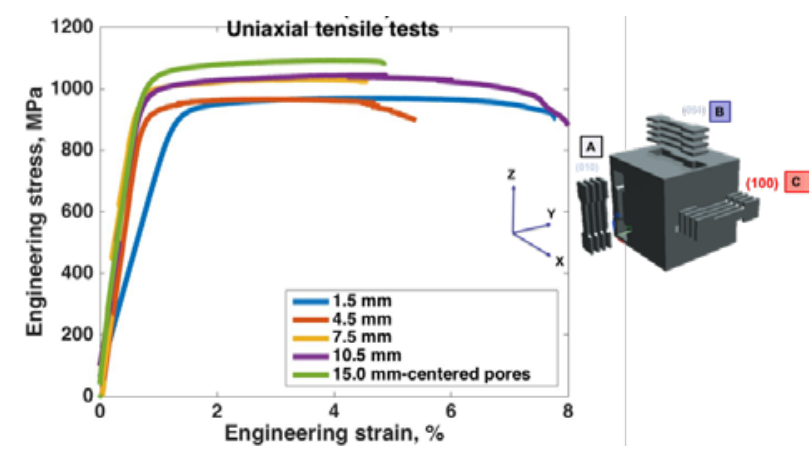

Figure 7:Tensile behavior of $800 \mathrm{~W}$ (100) specimens at various distances from cubic component surface

As the tensile specimens move toward the center of the cube, where the greatest re-melting occurs, there is an overall increase in yield strength and ultimate tensile strength (UTS). However, elongation values moving within the cube in the (100) direction do not show a steady trend. This could possibly be due to the placement of hatch-layer porosity, with pore alignment in the center of the tensile axis exhibiting less elongation than specimens with pore alignment closer to the surface of the tensile specimen. Pore alignment may also influence the Young's modulus as well.

\subsection{Influence of Tensile Specimen Orientation in the Cubic Component}

Based on the results in Section 4.1, the differences between each orientation within the $800 \mathrm{~W}$ processed cubic component are apparent. To determine whether porosity or phase composition dictated the difference in mechanical behavior for the three orientations, XRD analysis for each orientation, denoted "A" for (010), "B" for (100) and "C" for (001), was used to examine whether phase composition was a deciding factor, with the results in Figure 6.

The XRD pattern shows negligible differences in phase composition for each orientation, with similarities to conventionallyprocessed, annealed Ti6-Al-4V. The resulting XRD pattern shows that phase composition has little influence on the variations in mechanical behavior

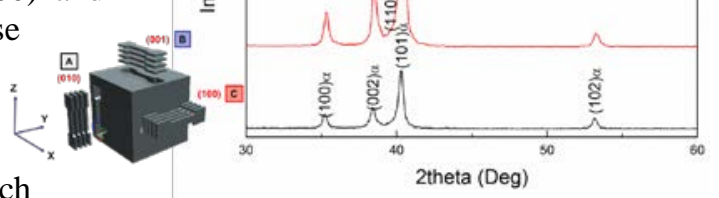

Figure 8: X-ray diffraction patterns for each orientation in the $800 \mathrm{~W}$ processed Ti-6Al-4V cubic for each orientation and is the motivation to analyze porous structure in-depth, assuming that porosity has the largest influence on mechanical behavior.

In addition to investigating the porosity of each tensile specimen, testing tensile specimens in LENS-processed Ti-6Al-4V cubic components processed with different laser powers were evaluated. Figure 7 shows the stress-strain curves for the $710 \mathrm{~W}$ processed cube in the (001) and (100) directions, and the $800 \mathrm{~W}$ processed cube in the (010), (001) and (100) directions. The $710 \mathrm{~W}$ cube exhibits the same trends - increase in elongation and strength in the (100) orientation, as well as an increase and slight embrittlement closer at greater distances from the surface of the cubic components. Though yield strength and UTS values are comparable between the $710 \mathrm{~W}$ and $800 \mathrm{~W}$ processed tensile specimens, the elongation increased with the increase in power. 

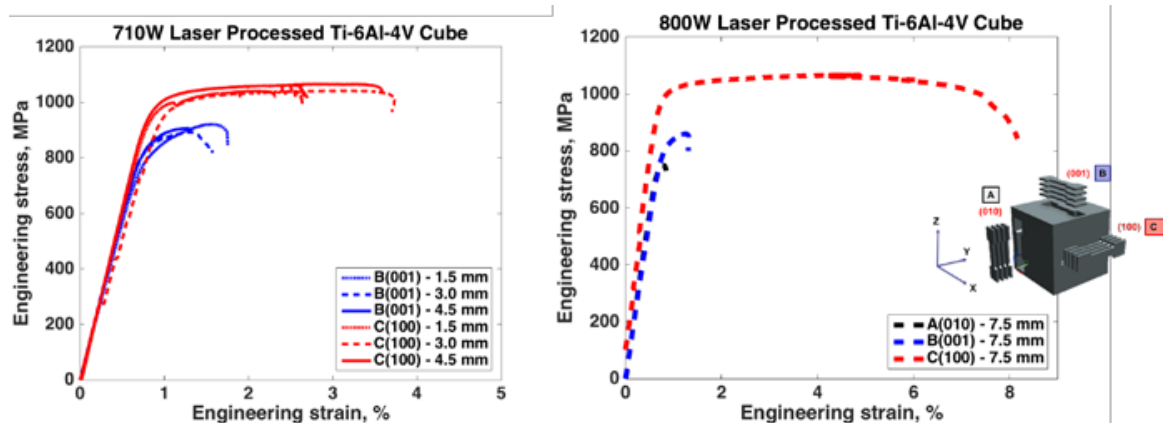

Figure 9: Influence of orientation on mechanical behavior for $710 \mathrm{~W}$ and $800 \mathrm{~W}$ cubic components

\subsection{Influence of Laser Processing Power}

For a more comprehensive evaluation of laser processing power, tensile specimens in (100) orientation in the $940 \mathrm{~W}$ processed cubic component were also tested. Figure 8 shows the mechanical behavior of tensile specimens in all three cubic components but only in the (100) orientation. Tensile specimens in the 940W cubic component reinforced the observation in Section 4.2, where elongation and strength increases with increased laser processing power. Tensile specimens in the $940 \mathrm{~W}$ cubic with (100) orientation exhibit consistency and show relatively similar elongation and strength values within a margin of error.

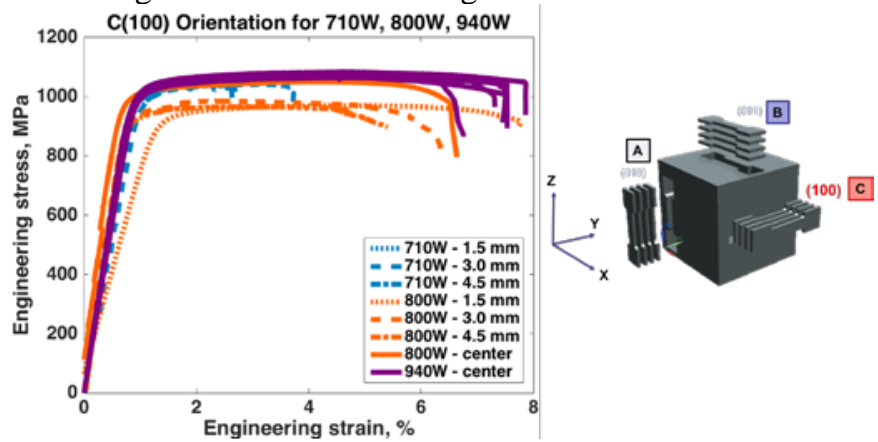

Figure 10:Influence of laser processing power on mechanical behavior in the (001) orientation

Fractography analysis of the fracture surface of representative tensile specimens were utilized to determine the mechanism of failure, whether it was elastic incompatibility, plastic deformation of crystalline solids, coalescence of plastic cavities, or boundary sliding. Figure 9 shows representative fracture surfaces for each processing process and orientation.

\begin{tabular}{|c|c|c|c|}
\hline & 710W & $800 W$ & 940W \\
\hline$A(010)$ & & Exers & \\
\hline
\end{tabular}




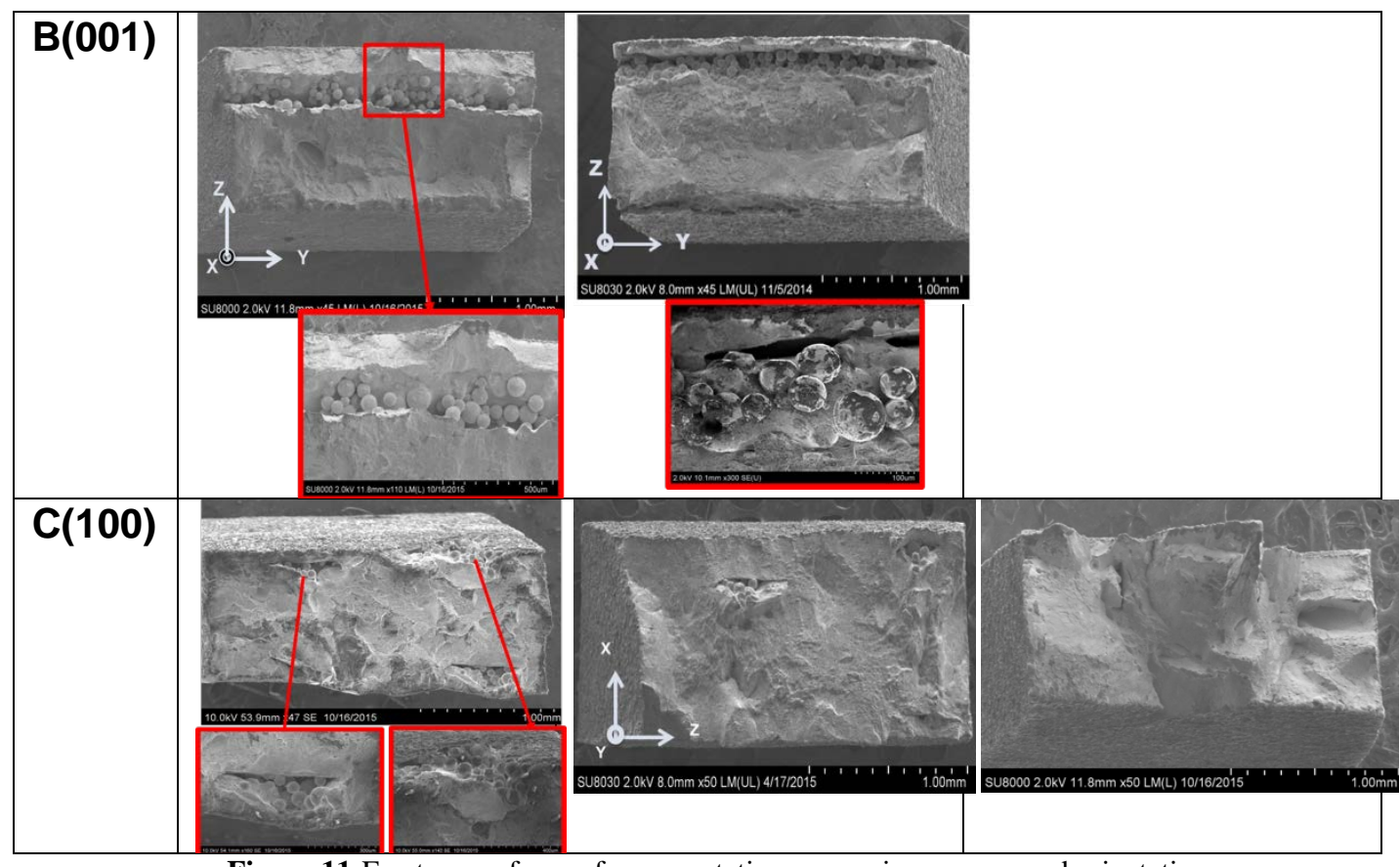

Figure 11:Fracture surfaces of representative processing powers and orientation.

For the fracture surfaces in the $\mathrm{A}(010)$ orientation, the fracture occurs at the interface of each layer with noticeable unmelted powders. River lines are apparent in both fracture surfaces and are characteristic of brittle fracture. However, the $800 \mathrm{~W}$ A(010) fracture surface exhibits more of a cupcone fracture, indicating that ductile fracture occurred.

For the fracture surfaces in the $\mathrm{B}(001)$ orientation, the fracture occurs at the interface of each hatch spacing, or overlap between scanning beads within the same layer. There are also noticeable unmelted powders that are aligned, indicating the location of the hatch overlap. Compared to $A(010)$ orientation, the surfaces exhibit more topography and dimples, which are characteristic of ductile fracture, with the 800W surface exhibiting more complex topography.

For the fracture surfaces in the $\mathrm{C}(100)$ orientation, there is a negligible amount of unmelted powders compared to the other orientations, and with a decrease in unmelted powders with an increase in laser processing power. In addition, an increase in processing power shows an increase in topography and cup-cone fracture, indicating an increase in ductile fracture.

By examining the fracture surfaces, the changes in elongation and strength with processing power and orientation observed during mechanical testing are validated. Relationships between the bulk porosity with each processing power and orientation and the resulting mechanical behavior can also be made. By plotting out this relationship in Figure 10, the slope of each data set indicates the degree of anisotropy in the orientation direction and processing power with $800 \mathrm{~W} A(010)$ exhibiting the greatest anisotropy, or greatest variation in mechanical behavior with a change in porosity. This means that changes in the microstructure, particularly porosity, in the $800 \mathrm{~W} \mathrm{~A}(010)$ tensile specimens greatly influence the mechanical properties as compared to $940 \mathrm{~W} \mathrm{C}(100)$ where there is little change in mechanical behavior even with a change in porosity. 


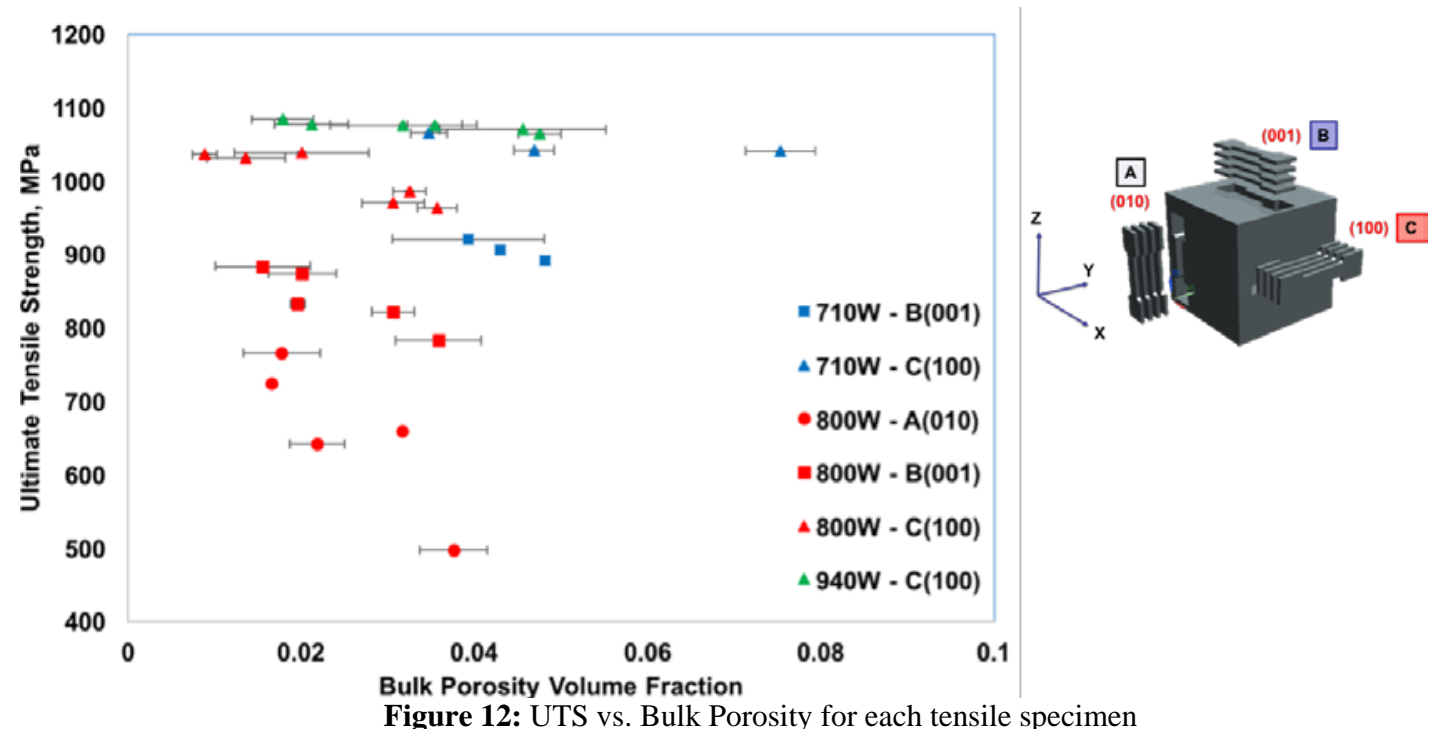

\section{J-Factor Analysis}

To determine how process parameters influence the J-factor and to determine the J-factor itself, 2D optical micrographs were captured for each tensile specimen, with each pore in the 2D micrographs investigated for their statistical distributions in roundness, circularity and their distances from one another. Figure 11 is an example of a binary 2D micrographs for a tensile specimen in the $C(100)$ orientation in the $940 \mathrm{~W}$ processed cubic component, located about $3.0 \mathrm{~mm}$ from the surface of the cube. Though the pores are aligned, there exit ranges of circularity, roundness and distribution. By using the approximation of the Taylor series in the GMR relationship in Eqn. 3 from Section 3, the Jfactor can be calculated for each data set by knowing the bulk porosity values and the UTS.

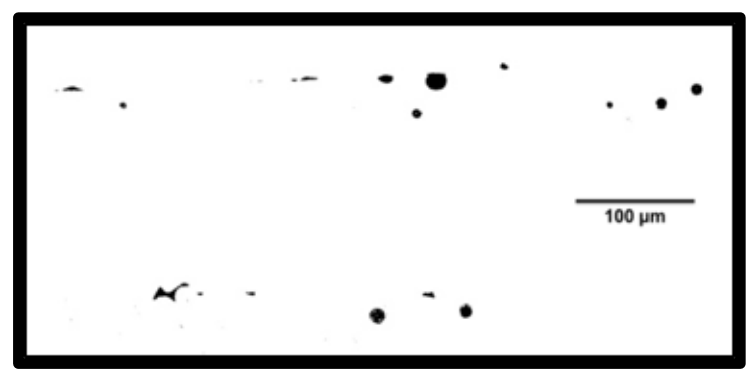

Figure 13: 2D Binary micrograph of a part of a tensile specimen in the $\mathrm{A}(010)$ orientation in the $800 \mathrm{~W}$ processed cube

By calculating these three independent variables for hundreds of pores for various tensile specimens in each power and orientation, probability distribution curves were determined for each variable to determine the $a, b, c$ dimensions of the ellipsoid pores in the various orientations. Based on the probability distributions, the mean values for $a, b$, and $c$ are $40 \mu \mathrm{m}, 20 \mu \mathrm{m}$ and $4 \mu \mathrm{m}$ respectively. By using values and the thermal strain parameters, unknown constants can be determined to solve for the J-factor of the various laser power and orientations, which is the inverse of the slopes of the porosity values in Figure 11. Overall, an in-depth investigation of 2D optical micrographs to make 
calculations on each pore can be enlightening as to how the size and shape of the pores vary with laser processing power, orientation and location within the cubic component in terms of distance from the surface. These relationships can possibly link process parameters and porosity.

\section{Conclusions}

This study aimed to present an in-depth experimental analysis of LENS-processed Ti-6Al-4V components built with various laser processing powers in order to understand the basis for achieving superior mechanical properties in AM components as compared to cast titanium alloys. Overall, the key conclusions from this paper include:

- This paper presented an experimental study on tensile behavior and location relative to the surface of the LENS-processed cubic component, orientation within the component, and with laser processing power.

- This paper showed the relationships between tensile behavior with the microstructure of the components, including phase composition analysis via X-ray diffraction, fractography analysis, and bulk porosity of the tensile specimens.

- This paper took a comprehensive look at individual pores in the tensile specimens that may have caused failure, including an investigation in the area and roundness, that will contribute to the GMR and J-factor analysis for predictability.

Future work includes an investigating at the solidification microstructure, including cellular and lamellar grains as well as the concentration of alpha and beta phases within the cubic components. These microstructure analyses would be compared with thermo-mechanical simulation results that show the gradient of temperatures and cooling rate throughout a AM-built component. Ultimately, a predictable model would be finalized and would be able to provide information about the microstructure and mechanical properties based on constant material properties and process parameters. This modelling effort would be beneficial to further additive manufacturing research.

\section{Acknowledgements}

This work is supported by the National Institute of Standards and Technology (NIST) through award number 70NANB13H194. This work made use of the OMM Facility supported by NSF(DMR 1121262). QCML built the Ti-6Al-4V components. The author would also like to thank Olivia Schneider and Jacob Smith from Northwestern University.

\section{References}

[1] Niinomi M. Mechanical properties of biomedical titanium alloys. Materials Science and Engineering: A1998; 243 (1-2), 231-236.

[2] Kobryn PA, Semiatin SL. Mechanical properties of laser-deposited Ti-6Al-4V. Solid Freeform Fabrication Proceedings 2001; Aug 6 (pp. 6-8). Austin.

[3] Standard, A. S. T. M. Standard terminology for additive manufacturing technologies. PA: ASTM International; 2012; West Conshohocken.

[4] Griffith ML, Keicher DM, Atwood CL, Romero JA, Smugeresky JE, Harwell LD, Greene DL. Free form fabrication of metallic components using laser engineered net shaping (LENS). Proceedings of the Solid Freeform Fabrication Symposium 1996 Aug 12 (pp. 125-131). Austin, TX: University of Texas at Austin. 
[5] Atwood C, Griffith ML, Schlienger ME, Harwell LD, Ensz MT, Keicher DM, Schlienger ME, Romero JA, Smugeresky JE. Laser engineered net shaping (LENS): a tool for direct fabrication of metal parts. Proceedings of ICALEO 1998 Nov 16 (Vol. 98, pp. 16-19).

[6] Gibson I, Rosen DW, Stucker B. Additive manufacturing technologies. New York: Springer; 2010. [7] Thijs L, Verhaeghe F, Craeghs T, Van Humbeeck J, Kruth JP. A study of the microstructural evolution during selective laser melting of Ti-6Al-4V. Acta Materialia. 2010 May 31;58(9):3303-12.

[8] Alcisto J, Enriquez A, Garcia H, Hinkson S, Steelman T, Silverman E, Valdovino P, Gigerenzer H, Foyos J, Ogren J, Dorey J. Tensile properties and microstructures of laser-formed Ti-6Al-4V. Journal of materials engineering and performance. 2011 Mar 1;20(2):203-12.

[9] Wang T, Zhu YY, Zhang SQ, Tang HB, Wang HM. Grain morphology evolution behavior of titanium alloy components during laser melting deposition additive manufacturing. Journal of Alloys and Compounds. 2015 May 25;632:505-13.

[10] Baufeld B, Van der Biest O, Gault R. Additive manufacturing of Ti-6Al-4V components by shaped metal deposition: microstructure and mechanical properties. Materials \& Design. 2010 Jun 30;31:S106-11.

[11] Bian L, Thompson SM, Shamsaei N. Mechanical Properties and Microstructural Features of Direct Laser-Deposited Ti-6Al-4V. JOM. 2015 Mar 1;67(3):629-38.

[12] Carroll BE, Palmer TA, Beese AM. Anisotropic tensile behavior of Ti-6Al-4V components fabricated with directed energy deposition additive manufacturing. Acta Materialia. 2015 Apr 1;87:309-20.

[13] Xu W, Brandt M, Sun S, Elambasseril J, Liu Q, Latham K, Xia K, Qian M. Additive manufacturing of strong and ductile Ti-6Al-4V by selective laser melting via in situ martensite decomposition. Acta Materialia. 2015 Feb 15;85:74-84.

[14] Standard, A. S. T. M.Standard Test Methods for Tension Testing of Metallic Materials. Annual Book of ASTM Standards; 2004.

[15] Ji S, Gu Q, Xia B. Porosity dependence of mechanical properties of solid materials. Journal of Materials Science. 2006 Mar 1;41(6):1757-68.

[16] Mukherjee T, Zuback JS, De A, DebRoy T. Printability of alloys for additive manufacturing. Scientific Reports. 2016 Jan 22;6:19717.

[17] Bird RB, Stewart WE, Lightfoot EN. Transport phenomena. 2002. JohnWiley\& Sons, New York. 